\title{
Observations of Comet 1884 III (Wolf)
}

on the Equatorial of Morrison Observatory, Glasgow, Missouri.

\section{By C.W. Pritchett.}

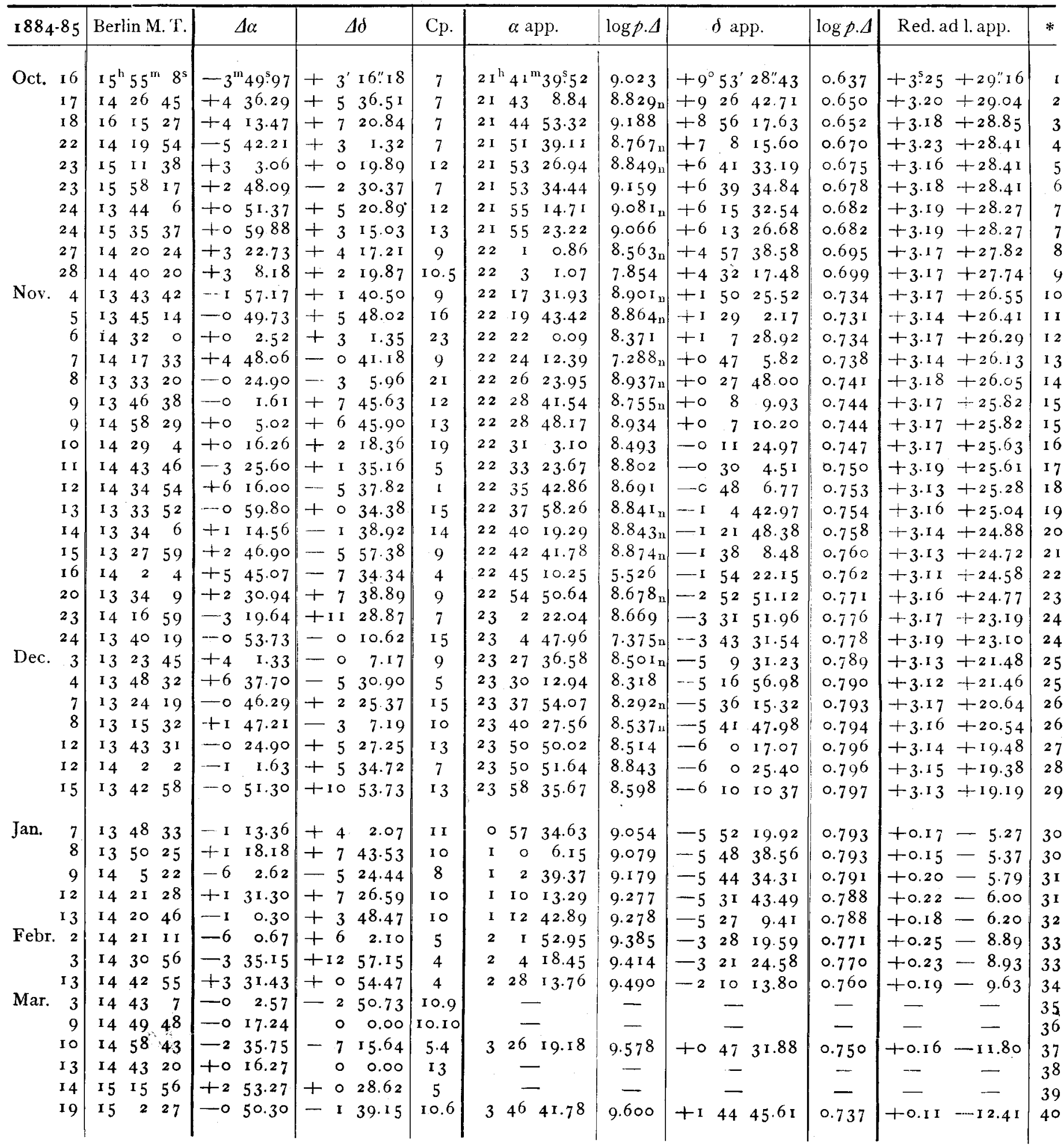


Adopted mean Places of Comparison Stars.

\begin{tabular}{|c|c|c|c|c|c|c|}
\hline$*$ & \multicolumn{3}{|c|}{$\alpha 1884.0$} & \multicolumn{2}{|c|}{$\delta$ I 884.0} & Authority \\
\hline 1 & $2 \mathrm{I}^{\mathrm{n}}$ & $45^{m}$ & 26.24 & $+9^{\circ}$ & $49^{\prime} 43^{\prime \prime} \circ 9$ & BB. VI $+9^{\circ} 49$ I 3 \\
\hline 2 & & $3^{8}$ & $29 \cdot 34$ & +92 & 2037.16 & $\varepsilon$ Pegasi. Am. Ephem. \\
\hline 3 & & 40 & 36.67 & +8 & $48 \quad 27.94$ & $\begin{array}{l}\text { Glasg. } 5573\left(W_{1} 2 \mathrm{r}^{\mathrm{h}} 946 \text {, }\right. \\
\left.\text { Lal. } 4^{2437}\right)\end{array}$ \\
\hline 4 & & 57 & I 8.09 & +7 & 445.87 & $\operatorname{Lam}_{2} 62$ I $8\left(W_{1} 2\right.$ I $\left.^{\mathrm{h}} 1296\right)$ \\
\hline 5 & & 50 & 20.72 & +64 & $40 \quad 44.89$ & BB. VI $+6^{\circ} 49^{2} 7$ \\
\hline 6 & & 50 & $43 \cdot 16$ & +64 & 4136.80 & $\begin{array}{l}\text { BB. VI }+6^{\circ} 493^{\circ} \\
\left(W_{1} 2^{\mathrm{h}}{ }^{\mathrm{L}} \mathbf{5}^{\circ}\right)\end{array}$ \\
\hline 7 & 2 I & 54 & 20.15 & +6 & $943 \cdot 3^{8}$ & $\begin{array}{l}1 / 2(\text { Y.9638+Schj } 8963) \\
\quad(\text { Arm. 4799) }\end{array}$ \\
\hline 8 & $2 \mathrm{I}$ & 57 & 34.96 & +4 & $5^{2} 53.55$ & Schj. 8995 (Lal. 43002) \\
\hline 9 & & 59 & $49 \cdot 7^{2}$ & +4 & $29 \quad 29.87$ & $v$ Pegasi, Glasg. 568 \\
\hline 10 & 22 & I 9 & 25.94 & +1 & $48 \quad 24.47$ & $\operatorname{Lam}_{1} 8824\left(W_{1} 2_{2}{ }^{\mathrm{h}} 380\right)$ \\
\hline I I & & 20 & $30.0 \mathrm{I}$ & +1 & $22 \quad 47.74$ & $\begin{array}{l}\text { Schj. 9172 }\left(W_{1} 22^{\mathrm{h}} 399,\right. \\
\text { Lal. } 43796)\end{array}$ \\
\hline I 2 & & $2 \mathrm{I}$ & $54.4^{\circ}$ & +1 & $4 \quad \mathrm{I} .28$ & $\operatorname{Lam}_{1} 8839\left(W_{1} 22^{\mathrm{h}} 437\right)$ \\
\hline I 3 & & 19 & 21.19 & +0 & $47 \quad 20.87$ & $\pi$ Aquarii. Am. Ephem. \\
\hline$x_{4}$ & 22 & 26 & 45.68 & +03 & 3027.91 & Schj. 9218 (Lal. 44036) \\
\hline 15 & & 28 & 39.98 & -0 & $\circ \quad I .52$ & $\begin{array}{l}1 / 4 \text { (BB.VI } 4383+\text { Glasg. } \\
5876+\text { Rii. I0377 } \\
\text { Arm. 4957) }\end{array}$ \\
\hline 16 & 22 & 30 & 43.66 & -0 & $148.9^{6}$ & $\begin{array}{l}1 / 2\left(B B . \text { VI } 43^{8} 7+\text { Schj. }\right. \\
\left.9^{2} 4^{6}\right)\end{array}$ \\
\hline 7 & 22 & $3^{6}$ & 46.08 & -0 & 5.48 & $5918+\mathrm{Sj} .929 \mathrm{I}-92)$ \\
\hline
\end{tabular}

\begin{tabular}{|c|c|c|c|}
\hline$\approx$ & a I $884-85.0$ & $d_{1} 884-85.0$ & Authority \\
\hline 18 & $22^{\mathrm{h}} 29^{\mathrm{m}} 23^{\mathrm{s}} \cdot 73$ & $-0^{\circ} 42^{\prime} 54 . .23$ & y Ayuarii. Am. Ephem. \\
\hline 19 & $\begin{array}{lll}22 & 38 & 54.9^{\circ}\end{array}$ & $\begin{array}{lll}\text {-I } & 5 & 42.39\end{array}$ & 9 fil. micr.comp. - Note \\
\hline 20 & $\begin{array}{lll}22 & 39 & 1.5^{8}\end{array}$ & $\begin{array}{lll}-1 & 20 & 34.34\end{array}$ & $1 / 2\left(G l .593^{\circ}+\right.$ Sj. 9307 \\
\hline 21 & $22 \quad 39 \quad 51.75$ & -I $32 \quad 35.82$ & Glasg. $593^{6}\left(W_{1} \mathbf{2}^{2 h}\right.$ \\
\hline 22 & 223922.06 & $\begin{array}{llll}-1 & 47 & 12.39\end{array}$ & Schj 93 I3 (Lal. 44 \\
\hline 23 & $22 \quad 52 \quad 16.54$ & $-3 \circ 54.78$ & $1 / 2$ (Gl.6or $3+$ Schj. \\
\hline 24 & ${ }^{2} 3 \quad 5 \quad 3^{8.5} \mathrm{I}$ & $\begin{array}{lll}-3 & 43 & 44.02\end{array}$ & Wash. Mer. Circ. - \\
\hline 25 & $2323 \quad 32.12$ & $\begin{array}{lll}-5 & 9 & 47.54\end{array}$ & $9 y$ r. 2204 \\
\hline 26 & $23 \quad 38 \quad 37 \times 19$ & $\begin{array}{lll}-5 & 39 & \mathbf{I} \cdot 33\end{array}$ & 7 fil. micr. comp. - Note \\
\hline 27 & $235 \mathrm{I}$ I I. 78 & $\begin{array}{lll}-6 & 6 & 3.80\end{array}$ & $W_{1} 23^{\mathrm{h}} 1016$ \\
\hline 28 & 235 I 50.12 & $\begin{array}{lll}-6 & 6 & 19.5^{\circ}\end{array}$ & $W_{1} 23^{\text {h }} 1029$ \\
\hline 29 & $23 \quad 592^{23.84}$ & $\begin{array}{lll}-6 & 2 \mathrm{I} & 23.29\end{array}$ & iscium. $9 y r .2259$ \\
\hline $3 \circ$ & $\begin{array}{lll}\circ & 587.82\end{array}$ & $\begin{array}{lll}-5 & 56 & 16.72\end{array}$ & I o fil micr comp. - Note \\
\hline $3 \mathbf{I}$ & I 841.79 & $\begin{array}{lll}-539 & 4.08\end{array}$ & $\begin{array}{r}\text { Schjell. } 396 \text { (Lal. } 2224 \\
\left.W_{1} \mathrm{r}^{\mathrm{h}}{ }_{9} 8\right)\end{array}$ \\
\hline 32 & I I $343.0 \mathrm{I}$ & -53051.70 & $\operatorname{schj.} 4$ I6 $\left(W_{1} I^{h_{1}} 8_{3}\right)$ \\
\hline 33 & $\begin{array}{lll}2 & 7 & 53.3\end{array}$ & 334 I 2.80 & $1 / 2$ (Stone $88 \mathrm{I}+\mathrm{Gl} .488$ \\
\hline 34 & $2 \quad 24 \quad 42.14$ & $-210 \quad 58.64$ & $\mathrm{~W}_{1} 2^{\mathrm{h}} 3^{8} 7$ \\
\hline 35 & $\ldots$ & - & DM. $-0^{\circ} 517$ \\
\hline $3^{6}$ & - & - & DM. $+0^{\circ} 600$ \\
\hline $\begin{array}{l}37 \\
38\end{array}$ & $\begin{array}{lll}3 \quad 28 & 54.77 \\
\end{array}$ & to $5459.3^{2}$ & $\begin{array}{l}\text { BB. VI }+0^{\circ} 600 \\
\text { Anonymous. }- \text { Note. }\end{array}$ \\
\hline 39 & - & $\ldots$ & DM. $+1^{\circ} 634$ \\
\hline 40 & $\begin{array}{lll}3 & 47 & 31.97\end{array}$ & $+146 \quad 37.27$ & Glasg. 9 I $4\left(W_{1} 3^{h} 888\right)$ \\
\hline
\end{tabular}

Notes.

No. I9 is $W_{1} 22^{\mathrm{h}} 790$, but was compared with Schj. $9358.59=$ Glasg. 5963. The differences corrected for Refraction being $\alpha^{\prime}-\alpha=-5^{\mathrm{m}} 50^{\mathrm{s}} .25, \delta^{\prime}-\delta=+5^{\prime} 49^{\prime \prime} 45$.

》24. By request, this star, which is $W_{1} 23^{\mathrm{h}} 5 \mathrm{I}$, was reobserved on the Meridian Circle at Washington, Nov. 29 and Dec. 3 and 4. Prof. Eastman gives the apparent place for Dec. 3, $\alpha=23^{\mathrm{h}} 5^{\mathrm{m}} 4^{\mathrm{s}} \cdot 55, \delta=-3^{\mathrm{o}} 43^{\prime} \mathbf{2} \mathrm{I}^{\prime \prime}$.60.

126. This star was compared with 795 Wash. Cat. of $1875=666$ Cat. of 1876 . The corrected differences were $a^{\prime}-\alpha=-3^{\mathrm{m}}$ I 5.0 I, $\delta^{\prime}-\delta^{\prime}=-4^{\prime} 33^{\prime \prime} .29$.

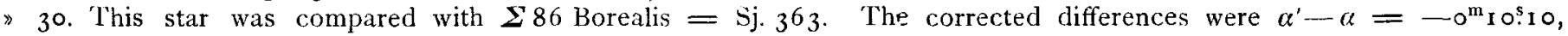
$\delta^{\prime}-\delta=+8{ }^{\prime} 6^{\prime \prime} .73$.

》 38. 'This star is Mag. 9.8 and is not in the Durchmusterung, it follows DM. $+\mathrm{I}^{\circ} 634$, nearly $20^{\mathrm{s}}$ and is $\mathrm{S}$ of it. No opportunity was afforded to obtain its place.

On Nov. 6, at $14^{\mathrm{h}} 29^{\mathrm{m}}$ Berlin M. T., the Comet exactly covered a star of Mag. 9.6, not given in the DM. The star shone with a pearly whiteness through the center of the Comet.

All the differences have been corrected for Refraction.

Observations on this Comet were delayed for some time on account of error in the Telegram, ${ }^{*}$ ) which assigned a daily motion Northward of $26^{\prime}$.

'The Comet was readily found from the Ephemeris, on April 3; it was then very faint, and observations with a Filar-micrometer were barely possible.

Morrison Observatory, Glasgow, Missouri, 1885 April г.

C. W. Pritchett.

*) Wegen dieses Irthums vergl. die Kemerkung in A. N. 2615 pag. 367.

$K r$. 